\title{
Gender Classification Based on Eye Movements: A Processing Effect During Passive Face Viewing
}

\author{
Negar Sammaknejad' ', Hamidreza Pouretemad ${ }^{2}$, Changiz Eslahchi', \\ Alireza Salahirad ${ }^{4}$, and Ashkan Alinejad ${ }^{5}$ \\ ${ }^{1}$ Institute for Cognitive and Brain Sciences, Shahid Beheshti University, Tehran, Iran \\ ${ }^{2}$ Department of Psychology \& Institute for Cognitive and Brain Sciences, Shahid Beheshti University, Tehran, Iran \\ ${ }^{3}$ Department of Computer Sciences, Shahid Beheshti University and Institute for Research in Fundamental Sciences, \\ Tehran, Iran \\ ${ }^{4}$ Department of Computer Sciences, University of South Carolina, Columbia \\ ${ }^{5}$ Department of Computer Sciences, University of Tehran, Tehran, Iran
}

ABSTRACT

Studies have revealed superior face recognition skills in females, partially due to their different eye movement strategies when encoding faces. In the current study, we utilized these slight but important differences and proposed a model that estimates the gender of the viewers and classifies them into two subgroups, males and females. An eye tracker recorded participant's eye movements while they viewed images of faces. Regions of interest (ROIs) were defined for each face. Results showed that the gender dissimilarity in eye movements was not due to differences in frequency of fixations in the ROls per se. Instead, it was caused by dissimilarity in saccade paths between the ROls. The difference enhanced when saccades were towards the eyes. Females showed significant increase in transitions from other ROls to the eyes. Consequently, the extraction of temporal transient information of saccade paths through a transition probability matrix, similar to a first order

Markov chain model, significantly improved the accuracy of the gender classification results.

gender classification

fixations, saccades,

Markov chain model,

left visual field bias

\section{INTRODUCTION}

Humans fixate more on more informative regions of a visual scene (Loftus \& Mackworth, 1978). When encoding faces, humans make more fixations to internal features such as eyes, nose, and mouth (Henderson, Williams, \& Falk, 2005; Stacey, Walker, \& Underwood, 2005), signifying the importance of these features in face processing (Walker-Smith, Gale, \& Findlay, 1977). Within the internal features, eyes are the most important components and receive the majority of fixations (Althoff \& Cohen 1999; Barton, Radcliffe, Cherkasova, Edelman, \& Intriligator, 2006; Henderson et al., 2005; Hickman, Firestone, Beck, \& Speer, 2010; Janik, Wellens, Goldberg, \& Dell'Osso, 1978). Moreover, despite other internal features (e.g., nose and cheek) that attract attention in frontal view, eyes are highly attended to regardless of the view (Sæther, Van Belle, Laeng, Brennen, \& Øvervoll, 2009). When viewing faces, about
$90 \%$ of the total fixation time is spent on internal features. About $60 \%$ of this fixation time is spent on and around the eyes (Henderson, Falk, Minut, Dyer, \& Mahadevan, 2001).

One of the factors affecting face processing is the sex of the observers (e.g., Hall, Hutton, \& Morgan, 2010; Heisz, Pattruff, \& Shore, 2013). Although males and females use similar scanning patterns when viewing faces, studies of eye movement have revealed subtle sex differences in attention to specific facial features. For instance, males make more fixations to the central region of a face and attend to nose and

Corresponding authors: Negar Sammaknejad, Institute for Cognitive and Brain Sciences, Shahid Beheshti University, Tehran, Iran. Phone: +98 (21) 29905406. E-mail:n_samaknejad@sbu.ac.ir 
cheeks while females attend to eye and brows regions (Sæther et al., 2009; Vassallo, Cooper, \& Douglas, 2009). Moreover, females attend to more detailed information when encoding faces, which results in their enhanced recognition ability (Heisz et al., 2013; Herlitz \& Rehnman, 2008).

Other than sex, the nature of the performed task may act as an exclusionary mechanism influencing gaze patterns, that is, eyes and lips are more informative during certain emotions and cause a shift in attention during emotion recognition tasks (Schurgin et al., 2014). For instance, more fixations are made to the eye region when participants look at angry faces and to the mouth region when they look at happy faces (Pérez-Moreno, Romero-Ferreiro, \& García-Gutiérrez, 2016). Likewise, observers fixate more on faces when they look at female faces compared to male faces. They especially focus more at the eye region of female faces than of male faces when they are asked to classify faces by gender (Armann \& Bülthoff, 2009; Pérez-Moreno et al., 2016).

In the present study, we attempted to answer two questions: (a) whether the differences that exist in the eye movement patterns of males and females are distinct enough to be utilized to estimate the sex of the viewers, and (b) whether the differences are task-independent and appear in a passive-viewing experiment.

Participants' eye movements were recorded while they passively viewed images of human faces. In terms of analysis, previous studies exploring eye movement data utilized a variety of methods. Coutrot, Hsiao, and Chan (2017) grouped these methods into four different approaches: (a) oculomotor (eye-movement) parameters, (b) spatial distributions of eye positions, (c) string-based and geometric scanpath comparisons, and (d) probabilistic approaches. Although the majority of previous studies exploring sex differences in face perception compared numbers or durations of fixations in the regions of interest (ROIs), some other studies that used more explorative analysis techniques, such as Hidden Markov Models (HMM), reached more precise results (Coutrot, Binetti, Harrison, Mareschal, \& Johnston, 2016). In the current study, our analysis falls in categories $a$ and $b$ of the mentioned approaches, meaning that in addition to parameters directly taken from the eye movement recordings, such as fixation locations or durations, saccade paths and fixation sequences were also included to investigate how frequently males and females shift attention between the ROIs. Using this probabilistic approach, we investigated whether it is possible to classify the gender of the viewers based on their eye movement patterns. Eight ROIs were defined for each face. Based on previous findings mentioned above, we expected that among these ROIs, females switch fixations between each ROI and eye regions and males switch fixations between each ROI and nose. We hypothesized that if the scanning patterns are influenced by sex (Heisz et al., 2013), a model based on participants' eye movements should be able to classify males and females into two subgroups. A comprehensive analysis was performed in three steps: For easier interpretation of the data, first, a proportion test that falls in the "Eye-movement direct parameters" approach was performed to compare the number of fixations in the ROIs between males and females. Then, a model was trained to classify the gender of the participants based on their fixation locations. At the end, a model based on the relative frequency of saccades between the ROIs, falling in the category of probabilistic approaches mentioned in the study by Coutrot et al. (2016), was utilized to train the model and distinguish subtle differences in the scanning patterns of males and females. The structure of this model was very similar to the transition probability matrix of a first order Markov chain model. The advantage of this model is that similar to an HMM, it contains temporal information as well as transitions (saccade patterns) between the ROIs. We show that the extracted temporal transition information of saccade paths accurately classified the gender of the participants.

\section{METHOD}

\section{Participants}

In total, 33 undergraduates, 18 females $\left(M_{\text {age }}=22.78\right.$ years, $\left.S D=2.96\right)$ and 15 males $\left(M_{\text {age }}=24.86\right.$ years, $\left.S D=4.39\right)$, from the Shahid Beheshti University participated in this study. All participants had normal or corrected-to-normal vision. Results from two additional participants were not included in the analysis due to their extensive head movement.

\section{Apparatus and Stimuli}

A remote desktop eye tracking device, SMI RED system (SensoMotoric Instruments), was used to monitor and record eye movement data. Stimuli were presented on a 22 in. monitor located approximately 50 $\mathrm{cm}$ from participants. iViewX (SensoMotoric Instruments) software was used to generate the experiment and BeGaze (SensoMotoric Instruments) software was used to collect and extract the eye movement data.

Stimuli consisted of 20 full-front face photos-10 males and 10 females-taken with a Canon 60D camera in high resolution. All photos were in color, were standardized for size and lighting, and portrayed neutral expression (see Figure 1). The people in the photos were unknown to the participants.

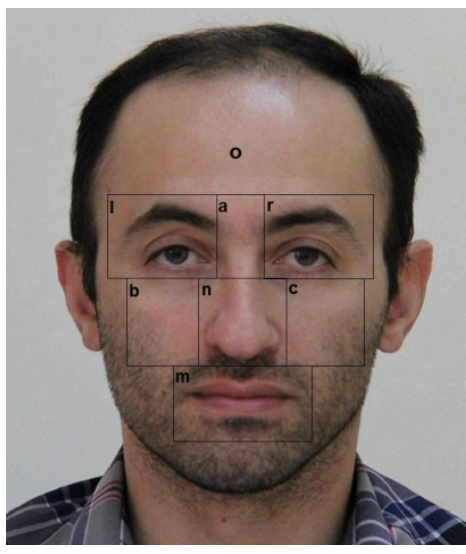

FIGURE 1.

An example of faces used in the experiment. Each face was divided into eight regions of interest (ROIs) identified as / (left eye), $a$ (between eyes), $r$ (right eye), $b$ (left cheek), $n$ (nose), $c$ (right cheek), $m$ (mouth), and $o$ (others). 


\section{Procedure}

The experiment took place in an eye-tracking laboratory with no ambient light other than the display. The procedure was divided into two phases: a calibration phase and a study phase. Calibration was included in the eye tracker software. Nine dots, one dot at a time, were presented at different positions on the monitor. Participants were asked to fixate and follow the dots. The process was repeated to validate the calibration. In the study phase, observers sat $50 \mathrm{~cm}$ from the monitor and read the instruction displayed on the screen. They were asked to passively view images of faces and were not informed about the goal of the experiment. Eye movements were sampled during the experiment at $120 \mathrm{~Hz}$. Each trial began with a fixation cross at the center of the screen for $750 \mathrm{~ms}$, followed by a face image that remained visible for 5 s. Faces were presented in a random order in two blocks separated by a 5 min break. Each face was shown five times. Thus, the experiment consisted of a total of 100 trials and approximately took $20 \mathrm{~min}$ for each participant.

\section{Regions of Interest}

To examine the data, eight ROIs were defined for each face. Data was processed to calculate the total number and duration of fixations in each ROI. The ROIs were defined as left eye $(l)$, between eyes $(a)$, right eye $(r)$, left cheek $(b)$, nose $(n)$, right cheek $(c)$, mouth $(m)$, and others $(o)$. The spatial coordination of fixations was mapped onto the ROIs. Figure 1 illustrates the ROIs defined for one of the faces used in the experiment.

\section{RESULTS}

\section{Fixation Patterns Across Facial Features}

A preanalysis of data showed a high correlation between the number and the duration of fixations. Thus, substituting one for the other did not change the results and the analysis was executed based on fixation numbers.

First, a proportion test was performed on the number of fixations over the five trials and was averaged across faces. This measure was used to examine whether the fixation proportions were different between males and females. Then, two statistical models were designed to classify males and females' eye movement data.

\section{Method 1. Statistical Analysis}

Average fixation numbers in each ROI were calculated across participants and images. About $79 \%$ of fixations were made to the internal ROIs $(l, a, r, b, n, c, m)$ and about $21 \%$ to the rest of the screen, defined as $o$. Thus, in the first part we excluded $o$ and restricted our analysis to the internal ROIs (see Figure 2, Panel A).

Dividing participants by sex, females made $14.33(S D=3.87)$ and males made $14.02(S D=4.27)$ fixations during the $5 \mathrm{~s}$ viewing period. Dividing faces by sex, females averaged 14.25 and 14.40 fixations and

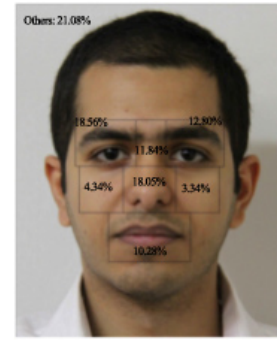

A

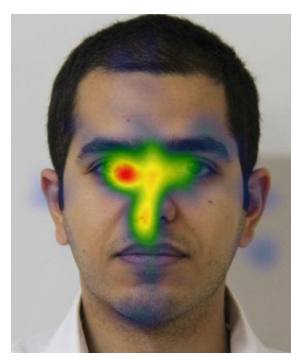

B
FIGURE 2.

Panel A: proportions of fixations in each region of interest (ROI). Only $21 \%$ of fixations were made to others (o). Panel B: heatmap of fixation counts. More fixations were made to the eye located in the left visual field (LVF).

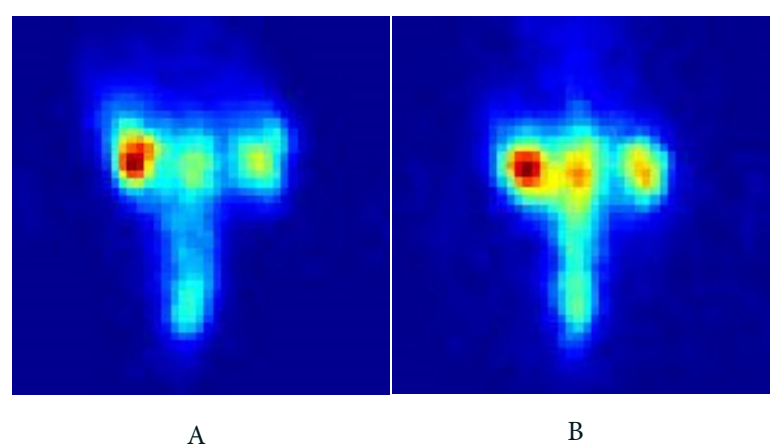

FIGURE 3.

Heatmaps of (A) female and (B) male participants on the average image obtained from all the images.

males averaged 14.08 and 13.96 fixations to male and female faces, respectively. To better visualize patterns of fixations, heatmaps were constructed with regard to the number of fixations (see Figure 2, Panel B). As Figure 2 illustrates, internal regions, especially the left eye (the eye that was located in the left visual field, LVF, of the viewer), were fixated the most.

An overall repeated-measures analysis of variance (ANOVA) showed no significant effect of sex, $F(1,31)=0.088, p=.769$. However, there was a significant effect of the ROIs, $F(8,31)=17.88, p<.001$. Figure 3 shows heatmaps of males and females displayed on the average of faces used in the experiment. On the one hand, as the figures illustrate, there are similarities and differences between the viewing patterns of males and females, that is, although both sexes fixated the most on the eye regions, females made more fixations to the eye regions than males did. On the other hand, males fixated at the center of the face (nose and between the eyes) slightly more than females and females fixated on the mouth slightly more than males. Average number of fixations in each ROI was calculated and compared between male and female participants. A $t$ test comparison showed that females made more fixations to the eye region than males $\mathrm{did}, t(31)=1.61, p=$ .039. Also, although the result was not significant, the data shows that on average, females made more fixations when they looked at female faces $(3.159, S D=1.82)$ than male faces $(2.68, S D=1.90)$. 


\section{Method 2. Fixation Locations and Fixation Transitions}

The hypothesis for doing this analysis was that if males and females have different viewing patterns while looking at faces, it should be possible to predict their sex based on their eye movements.

\section{RELATIVE FREQUENCY OF FIXATION NUMBERS (RFFN)}

To test the hypothesis, first, we examined whether the differences that exist in fixation locations between males and females were distinct enough to be used for estimating the sex of the participants. As mentioned above, fixation locations were mapped onto the ROIs. Thus, a sequence of letters, each letter representing a ROI, was obtained for each trial. For instance, considering the following sequence, each $\mathrm{X}$ represents the horizontal location and each Y represents the vertical location of a fixation.

$$
(x, y)=(425,430),(291,921),(358,1671),
$$

$$
(233,2080),(199,2288),(116,2421)
$$

The eye tracker sampled the position of the eyes with a sampling rate of $120 \mathrm{~Hz}$, which results in 120 gaze points per second. The gaze points that were close in both time and space created one fixation point. Thus, each fixation indicates that the subject has stayed in a specific $x$ and $y$ location for a specific period of time (a fixation duration is about 200-350 ms) while the device was tracking every $8 \mathrm{~ms}$. After mapping the $x$ and $y$ locations onto the ROIs, the following sequence is obtained for that trial:

\section{amnnma}

Consequently, including the data acquired from all participants, two datasets were created: one for females, containing 1,800 sequences; and another one for males, containing 1,500 sequences. Using the sequences, the relative frequency of fixations in each ROI was calculated for each group, $P_{\mathrm{i}}=\frac{f_{\mathrm{i}}}{N}$, where $f_{\mathrm{i}}$ is the frequency of fixations in the $\mathrm{ROI}_{\mathrm{i}}$ and $N$ is the total number of fixations. Table 1 shows the two $1 \times$ 8 vectors computed for males and females, where each cell represents the probability of fixations in a ROI. Finally, it was examined whether the relative frequency of fixations in each ROI is an ideal estimator for identifying the sex of the participants.

\section{LEAVE-ONE-OUT METHOD}

A leave-one-out cross-validation method was used to train and fit the model and to test the dataset. Data from $N-1$ participants, where $N$ is the total number of participants, was used to train, and data from one participant (e.g., Participant 1) was used to test the dataset. Based on the trained data, the probability vectors were calculated for both groups. Then, the probability in which the sequences obtained from each participant belonged to each group was calculated. For example, considering a sequence of fixations such as llmonr, Equation (2) calculates the probability of fixations falling in each ROI:

$$
P(\text { llmonr })=P(l) * P(l) * P(m) * P(o) * P(n) * P(r)
$$

where Equation 2 is obtained based on the independent and identically distributed (i.i.d) assumption of observations. Note that the ANOVA and $t$ test performed in previous sections were also developed based on similar assumptions.

Using males and females' probability vectors, the probability of the sequence belonging to each group was calculated:

$$
\begin{aligned}
P(\text { llmonr }) \text { female }= & .2011 \times .2011 \times .0979 \times .2132 \times \\
& \times .1643 \times .1324=.00001836 \\
P(\text { llmonr }) \text { male }= & .1732 \times .1732 \times .1024 \times .2245 \times \\
& \times .1788 \times .1059=.00001306
\end{aligned}
$$

\begin{tabular}{|c|c|c|c|c|c|c|c|c|}
\hline ROIs & $l$ & $a$ & $r$ & $b$ & $n$ & $c$ & $m$ & $o$ \\
\hline $\begin{array}{l}\text { Fixation Probability } \\
\text { (Females) }\end{array}$ & .2011 & .1182 & .1324 & .0371 & .1643 & .0353 & .0979 & .2132 \\
\hline \multicolumn{9}{|c|}{ A } \\
\hline ROIs & $l$ & $a$ & $r$ & $b$ & $n$ & $c$ & $m$ & $o$ \\
\hline $\begin{array}{l}\text { Fixation Probability } \\
\text { (Males) }\end{array}$ & .1732 & .1323 & .1059 & .0436 & .1788 & .0389 & .1024 & .2245 \\
\hline \multicolumn{9}{|c|}{ B } \\
\hline
\end{tabular}

\section{TABLE 1.}

Females (A) and Males (B) Probability Vectors

Note. $\mathrm{ROI}=$ region of interest. Each face is divided into eight ROIs. These ROIs are identified as $l$ (left eye), $a$ (between eyes), $r$ (right eye), $b$ (left cheek), $n$ (nose), $c$ (right cheek), $m$ (mouth), and $o$ (others). Each cell represents the probability of fixations in a ROI.

\section{TABLE 2.}

Absolute Difference Between Males and Females Probability Vectors as a Function of Regions of Interest

\begin{tabular}{c|c|c|c|ccccc}
\hline ROIs & $l$ & $a$ & $r$ & $b$ & $n$ & $c$ & $m$ & $o$ \\
\hline $\begin{array}{c}\text { Absolute Difference in } \\
\text { Probabilities }\end{array}$ & .0279 & .0141 & .0264 & .0064 & .0144 & .0036 & .0044 & .0112 \\
\hline
\end{tabular}

Note. ROI $=$ region of interest. Each face is divided into eight ROIs. These ROIs are identified as $l$ (left eye), $a$ (between eyes), $r$ (right eye), $b$ (left cheek), $n$ (nose), $c$ (right cheek), $m$ (mouth), and $o$ (others). Each cell represents the probability of fixations in a ROI. The highest dissimilarities were obtained when participants fixated on the eye regions (highlighted in red). 


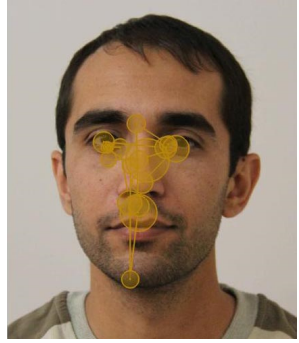

FIGURE 4.

The scanning pattern of a participant when viewing one of the faces.

The sequence belonged to the females group if the probability of its fixations falling in the ROIs was higher using the females vector ( $P$ female $>P$ male $)$ and belonged to the males group otherwise. Hence, considering all the sequences obtained from Participant 1 , the participant was recognized as female if the acquired probability ( $P$ female $_{\text {total }}$ ) was higher than $50 \%$ and vice versa. Calculating the probability of sequences, results showed that the probability of fixation locations could not accurately classify the gender of the participants.

A $t$ test comparison showed no significant difference between the probability vectors of males and females, $t(7)=-0.002, p=.998$. However, the highlighted columns in Table 2 indicate that the dissimilarities were the highest when participants fixated on the left and the right eyes.

\section{RELATIVE FREQUENCY OF SACCADE PATHS (RFSP)}

The first approach showed that the fixation counts in the ROIs were not sufficient for sex classification. Thus, in the second approach, saccades were also included in the analysis and a model based on the relative frequency of saccade paths (RFSP) was trained for each sex. The RFSP was calculated based on the frequency of saccades between each two ROIs divided by the total number of saccades. Figure 4 shows fixations and saccades made by one of the participants while viewing one of the faces. Circles represent fixations and the lines between them are the saccades.

Assuming independency in saccade paths, the likelihood of transiting from one ROI to another was calculated for males and females and a transition matrix was obtained for each group. Here, $p_{\mathrm{ij}}$ denotes the frequency of transition from region $i$ to $j$ divided by the total possible transitions. The probability of transitions from one ROI to another is summarized in two transition matrices (see Table 3).

One can compare the transition probabilities of Table 3 with the transition probability matrix of a first order Markov chain model (Kemeny \& Snell, 1960). Parameters of a Markov chain model can be obtained through a maximum likelihood estimation, that is,

$$
p_{\mathrm{ij}}=\frac{C_{\mathrm{ij}}}{C_{\mathrm{i}}}
$$

\section{TABLE 3.}

Females' (A) and Males' (B) Transition Matrices

\begin{tabular}{|c|c|c|c|c|c|c|c|c|c|}
\hline & & \multicolumn{8}{|c|}{ Target ROI } \\
\hline \multirow{9}{*}{$\begin{array}{c}\text { Source } \\
\text { ROI }\end{array}$} & Region & $l$ & $a$ & $r$ & $b$ & $n$ & $c$ & $m$ & $o$ \\
\hline & $l$ & .07449 & .01556 & .04447 & .00772 & .01962 & .00390 & .00886 & .02697 \\
\hline & $a$ & .03174 & .02644 & .02254 & .00229 & .01901 & .00143 & .00607 & .01084 \\
\hline & $r$ & .04373 & .01116 & .03601 & .00205 & .01166 & .00431 & .00821 & .01568 \\
\hline & $b$ & .00603 & .00139 & .00197 & .00702 & .00702 & .00209 & .00554 & .00451 \\
\hline & $n$ & .02114 & .01445 & .01404 & .01038 & .05461 & .01240 & .02874 & .01055 \\
\hline & $c$ & .00443 & .00114 & .00476 & .00295 & .00628 & .00591 & .00587 & .00336 \\
\hline & $m$ & .00763 & .00579 & .00529 & .00377 & .02513 & .00488 & .03178 & .01297 \\
\hline & $o$ & .02188 & .01305 & .01182 & .00344 & .01535 & .00287 & .008253 & .13444 \\
\hline \multicolumn{10}{|c|}{ A } \\
\hline \multirow{9}{*}{$\begin{array}{c}\text { Source } \\
\text { ROI }\end{array}$} & Region & $l$ & $a$ & $r$ & $b$ & $n$ & $c$ & $m$ & $o$ \\
\hline & $l$ & .05278 & .01522 & .02401 & .00794 & .01589 & .00337 & .00910 & .02206 \\
\hline & $a$ & .02685 & .02681 & .01416 & .00239 & .02015 & .00226 & .00510 & .01229 \\
\hline & $r$ & .01868 & .00812 & .02450 & .00253 & .01127 & .00479 & .00736 & .01451 \\
\hline & $b$ & .00683 & .00164 & .00266 & .00696 & .00683 & .00217 & .00421 & .00546 \\
\hline & $n$ & .02237 & .01265 & .01185 & .00941 & .05531 & .00887 & .02379 & .01056 \\
\hline & $c$ & .00368 & .00164 & .00350 & .00279 & .00630 & .00661 & .00359 & .00488 \\
\hline & $m$ & .00949 & .00430 & .00701 & .00275 & .01549 & .00377 & .02947 & .01606 \\
\hline & $o$ & .01677 & .01296 & .01056 & .00554 & .01584 & .00408 & .01287 & .12296 \\
\hline
\end{tabular}

Note. ROI $=$ region of interest. Each cell represents the relative probability of transition from one ROI to another; ROIs are identified as $l$ (left eye), $a$ (between eyes), $r$ (right eye), $b$ (left cheek), $n$ (nose), $c$ (right cheek), $m$ (mouth), and $o$ (others). The probabilities are color-coded. The highest transition probability is shown in red, the second highest is in orange, and the third highest is in yellow. 
where $C_{\mathrm{ij}}$ indicates the number of transitions from state $\mathrm{i}$ to $\mathrm{j}$, and $C_{\mathrm{i}}$ is the total number of transitions in state $\mathrm{i}$.

Although the proposed method of this article to obtain the total number of transitions $\left(C_{\mathrm{i}}\right)$ is different from a conventional Markov chain model, the core idea is still the same. Consequently, using this novel structure, the temporal information relevant to saccade paths is extracted. It is shown that the presence of such important auxiliary information significantly improves gender classification results.

As shown in the tables, excluding the other to other transition, the highest probability of transitions was obtained for transition from left eye to left eye (.075) for females and from nose to nose (.055) for males. The second highest probability was from nose to nose (.054) for females and from left eye to left eye (.053) for males, and the third highest was from left eye to right eye (.044) for females and from mouth to mouth (.029) for males.

Again, the leave-one-out method was applied and the probability of each sequence belonging to each sex category was calculated. For instance, considering a sequence such as llmonr for Participant 1, the probability of saccade paths was calculated with regard to both transition matrices:

$P(l l m o n r)=P(l l) * P(l m) * P(m o) * P(o n) * P(n r)$

\section{TABLE 4.}

Percentage Correct of the Estimated Sex

\begin{tabular}{|c|c|c|c|}
\hline $\begin{array}{c}\text { Female } \\
\text { Participants }\end{array}$ & $\begin{array}{c}\% \text { Correct } \\
\text { Estimations }\end{array}$ & $\begin{array}{c}\text { Male } \\
\text { Participants }\end{array}$ & $\begin{array}{l}\text { \% Correct } \\
\text { Estimations }\end{array}$ \\
\hline $\mathrm{F} 1$ & 45 & M1 & 87 \\
\hline $\mathrm{F} 2$ & 90 & M2 & 96 \\
\hline F3 & 85 & M3 & 93 \\
\hline $\mathrm{F} 4$ & 93 & M4 & 69 \\
\hline F5 & 72 & M5 & 51 \\
\hline F6 & 100 & M6 & 100 \\
\hline F7 & 97 & M7 & 99 \\
\hline F8 & 98 & M8 & 93 \\
\hline F9 & 91 & M9 & 52 \\
\hline F10 & 89 & M10 & 87 \\
\hline F11 & 97 & M11 & 98 \\
\hline F12 & 92 & M12 & 86 \\
\hline F13 & 97 & M13 & 92 \\
\hline F14 & 81 & M14 & 67 \\
\hline F15 & 93 & M15 & 85 \\
\hline F16 & 97 & & \\
\hline F17 & 100 & & \\
\hline F18 & 99 & & \\
\hline Average & 89.7 & Average & 83.6 \\
\hline
\end{tabular}

Note. On average, the percentage correct was $89.7 \%$ and $83.6 \%$ for female and male participants, respectively. where Equation 6 was obtained based on the properties of a first order Markov chain model without consideration of the prior information (Rabiner, 1989), that is,

$$
P(l l m o n r)=P(l \mid l) * P(l \mid m) * P(m \mid o) * P(o \mid n) * P(n \mid r)
$$

Therefore,

$$
\begin{aligned}
P(\text { llmonr }) \text { female }= & .7449 \times .00886 \times .01297 \times \\
& \times .01535 \times .01404=1.844791 \mathrm{e}-9 \\
P(\text { llmonr }) \text { male }= & .05278 \times .00910 \times .01606 \times
\end{aligned}
$$

$$
\times .1584 \times .011852=1.448115 \mathrm{e}-9
$$

Thus, a sequence belonged to the females' category if the probability of its saccade paths was higher using the females' matrix and vice versa. It is important to note that in Equations 6 and 7, the initial state distribution is considered to be uniform (with equal probabilities for states) which makes the comparison study independent of the initial state distribution. Table 4 shows the percentage of correctly estimated sex for male and female participants. For instance, considering the participant Female 1 (F1), 45\% of the sequences were correctly estimated as female. For each participant, the estimated sex was associated with the matrix that resulted in a percentage correct higher than $50 \%$. As shown in the table, the total percentage of correctly estimated sex of the participants was $94.7 \%$ for females and $100 \%$ for males; the estimation was wrong only for one female (F1). On average, the model correctly estimated the sex of the participants $89.7 \%$ and $83.6 \%$ of the time for females and males, respectively. As previously stated, this significant improvement in comparison to the relative frequency of fixation numbers (RFFN) method is due to the consideration of temporal transition information of saccade paths through the properties of a first order Markov chain model.

Comparing the two matrices. Paired-sample $t$ test showed a significant difference between the first and third columns of the two transition

\begin{tabular}{|c|c|c|c|c|c|c|c|c|c|}
\hline & \multicolumn{9}{|c|}{ Target ROI } \\
\hline \multirow{9}{*}{$\begin{array}{c}\text { Source } \\
\text { ROI }\end{array}$} & Regions & $l$ & $a$ & $r$ & $b$ & $n$ & $c$ & $m$ & $o$ \\
\hline & $l$ & + & + & + & - & + & + & - & + \\
\hline & $a$ & + & - & + & - & - & - & + & - \\
\hline & $r$ & + & + & + & - & + & - & + & + \\
\hline & $b$ & - & - & - & + & + & - & + & - \\
\hline & $n$ & - & + & + & + & - & + & + & - \\
\hline & $c$ & + & - & + & + & - & - & + & - \\
\hline & $m$ & - & + & - & + & + & + & + & - \\
\hline & $o$ & + & + & + & - & - & - & - & + \\
\hline
\end{tabular}
matrices. These two columns represent the probability of transitions

\section{TABLE 5A.}

Difference Matrix Illustrates the Sign of the Difference Between Transition Matrices of Males and Females.

Note. ROI $=$ region of interest. Regions of interest are identified as $l$ (left eye), $a$ (between eyes), $r$ (right eye), $b$ (left cheek), $n$ (nose), $c$ (right cheek), $m$ (mouth), and $o$ (others).A positive value (+) indicates that the probability of transition between the two regions of interest (ROIs) was higher for female participants and vice versa. Cells highlighted in dark gray indicate the max difference ( $l$ to $l, l$ to $r, r$ to $l$, and $r$ to $r$ ). 
TABLE 5B.

The Euclidean Distance Vector

\begin{tabular}{ccccccccc}
\hline ROIs & $l$ & $a$ & $r$ & $b$ & $n$ & $c$ & $m$ & $o$ \\
\hline Euclidean Distance & .034 & .0039 & .0252 & .0026 & .0104 & .0041 & .0077 & .0131 \\
\hline
\end{tabular}

Note. ROI $=$ region of interest. Regions of interest are identified as $l$ (left eye), $a$ (between eyes), $r$ (right eye), $b$ (left cheek), $n$ (nose), $c$ (right cheek), $\mathrm{m}$ (mouth), and $o$ (others). A positive value $(+)$ indicates that the probability of transition between the two regions of interest (ROIs) was higher for female participants and vice versa. Cells highlighted in dark gray indicate the max difference ( $l$ to $l, l$ to $r, r$ to $l$, and $r$ to $r$ ).

from other ROIs to the left and the right eyes. The probabilities were significantly higher for female participants than for male participants, $t(7)=1.778, p=.044$ for transitions to the left eye, $t(7)=1.982, p=.042$ for transitions to the right eye.

The difference and the Euclidean distance. The difference matrix, an $8 \times 8$ matrix representing the difference between the two matrices, was also calculated (see Table 5, Part A). Positive values indicate that the probability of transitions between the ROIs was higher for female participants and vice versa. Highlighted cells specify higher positive values showing that the probability of transitions between the two eyes was the highest for female participants.

We also computed the Euclidean distance vector. The Euclidean distance considered here is the distance between the respective columns, interpreted as vectors, of the male and female matrices. Part B of Table 5 indicates that the maximum differences between the two tran-

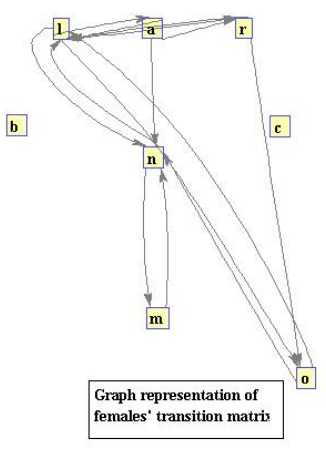

A

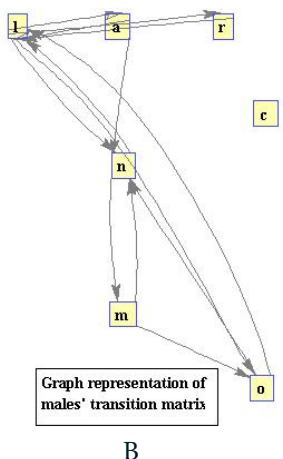

FIGURE 5.

Graph representations of females' (A) and males' (B) transition matrices. The lines between the regions of interest (ROIs) represent the saccade paths.

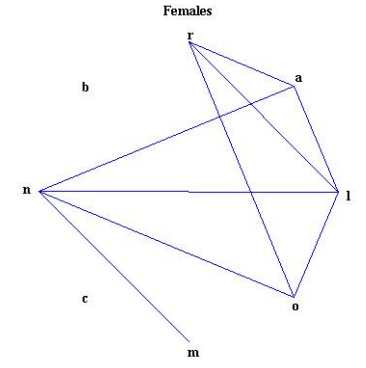

A

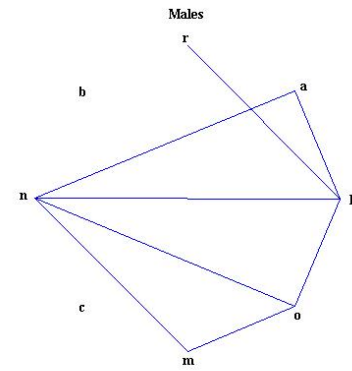

B
FIGURE 6

Nose and left eye are the most viewed regions and can be considered the two hubs of the system for both female (A) and male (B) participants. sition matrices were related to the left (.0305) and the right eye (.0252). Equation 10 was used to calculate the Euclidean matrix where $A$ and $B$ represent the transition matrices and $n$ is the number of the regions.

$$
d E(A, B)=\|A-B\|=\sum_{k=1}^{n}\left[\left(a_{k}-b_{k}\right)^{2}\right]^{\frac{1}{2}}
$$

\section{GRAPH REPRESENTATION OF THE TRANSITION MATRI- CES}

To better visualize the data, the transition matrices were transformed into graph representations. In the graphs, each node (state in a Markov chain model) represents a ROI and the lines between the nodes represent the saccade paths (see Figures 5 and 6). A threshold of .014 was applied to the transition probabilities, so transitions below that threshold did not appear in the graphs. On the one hand, as both figures show, there were similarities and differences between the viewing patterns of males and females. For instance, left eye and nose were highly connected regions when both males and females viewed faces, and hence, can be considered the hubs of the system. On the other hand, the right eye was viewed more by females and the mouth was viewed more by males.

\section{DISCUSSION}

We examined whether the sex of the observers influences their eye movement patterns when passively viewing images of faces, and furthermore, whether the difference is distinct enough to be used for sex classification. Eight ROIs were defined for each face and the number of fixations in each ROI was compared between males and females. Consistent with previous findings, our data showed that both males and females fixated more on the internal features, especially on the eyes, indicating the critical role of these features in face processing (Althoff \& Cohen, 1999; Barton et al., 2006; Henderson et al., 2001, 2005; Janik et al., 1978; Pérez-Moreno et al., 2016; Stacey et al., 2005). Although the gender difference in fixation counts in the ROIs was not significant, similar patterns as those of Heisz et al. (2013) showed that females exhibited more fixations to faces than males did.

Looking further into the data, we found different patterns of transitions (saccade) between the ROIs among males and females. Despite fixation locations, the difference in saccades was bold enough that a model based on the probability of transitions between the ROIs could accurately estimate the sex of the participants. Hence, the significant improvement of gender classification results were due to the consideration of temporal transition information of saccade paths through a transition probability matrix similar to its corresponding first order Markov chain model. 


\section{Fixation Numbers}

\section{PASSIVE- VERSUS TASK-ORIENTED VIEWING}

The lack of a significant difference in number of fixations between males and females was perhaps due to the nature of the experiment. The current experiment required passive viewing, with no additional task involved. Thus, participants could make a general sweeping scan of faces and did not need to attend more to features that are critical in face recognition tasks (Heisz et al., 2013) or in categorization tasks including gender and expression (Pérez-Moreno et al., 2016).

\section{The Mathematical Model}

In the second analysis approach, fixation locations and transitions between them were analyzed. Data showed that the saccadic eye movements were quite distinct among sexes and could be classified into two subpatterns; one for males and the other for females. Consequently, a model similar to its corresponding first order Markov chain structure, accurately estimated the sex of the participants based on their saccade paths. The comparison between males and females' transition matrices showed that the difference between saccade paths was mostly due to transitions from other ROIs to the eye region.

The influence of sex in various cognitive tasks (Halpern, 2000; Hamann, Herman, Nolan, \& Wallen, 2004; Hojjat, 2000; Stumpf, 1995; Voyer, Voyer, \& Bryden, 1995), especially in visual scanning patterns (Heisz et al., 2013; Hewig, Trippe, Hecht, \& Miltner, 2008), has been a subject of interest and drawn attention from a variety of disciplines and studies. In a sex identification task, although both males and females mostly focused on the central area of faces (Sæther et al., 2009), there were dissimilarities between their fixation patterns, that is, females attended more to the eyes and brows and males to the nose and cheeks. Our results showed that eye movement patterns of males and females were different even during a passive-viewing task, and similar to taskoriented studies, the difference was maximized when females looked at the eye region. The difference is clearly shown in the graph representations of transition matrices, that is, females made more transitions towards the eyes and males made more transitions towards the nose. Our results are consistent with those of Coutrot et al. (2016). They modeled the scanpaths of a large sample size (405 participants, 58 nationalities) while participants freely viewed videos of neutral faces. An HMM approach was used to train a gender classifier model based on discriminant analysis. Their data showed that both observer gender and gender of the faces being observed affect fixation patterns during face viewing. Females explored faces more than males did and showed a larger left-eye bias when looking at female faces. In the experiment presented in this paper, although the result for females' left-eye bias to female faces was not significant, similar to Coutrot et al's (2016) study, our data showed that females tended to fixate more at the left eye when viewing female faces compared to male faces. Moreover, males fixated less broadly and focused on the center of the face (between the eyes and nose regions) slightly more than females did.

The results emphasize that more accurate face recognition performance by females is perhaps due to their increased attention towards the eye (e.g., Hall et al., 2010; Herlitz \& Lovén, 2013).

\section{ACKNOWLEDGEMENTS}

We thank Ruhollah Mansouri for help with collecting data and Zahra Rezvani for help in programming and creating the graphs.

\section{REFERENCES}

Althoff, R. R., \& Cohen, N. J. (1999). Eye-movement-based memory effect: A reprocessing effect in face perception. Journal of Experimental Psychology: Learning, Memory, and Cognition, 25, 997-1010. doi: 10.1037//0278-7393.25.4.997 WwW

Armann, R., \& Bülthoff, I. (2009). Gaze behavior in face comparison: The roles of sex, task, and symmetry. Attention, Perception, \& Psychophysics, 71, 1107-1126. doi: 10.3758/APP.71.5.1107 WWW

Barton, J. J., Radcliffe, N., Cherkasova, M. V., Edelman, J., \& Intriligator, J. M. (2006). Information processing during face recognition: The effects of familiarity, inversion, and morphing on scanning fixations. Perception, 35, 1089-1105. doi: 10.1068/ p5547 WWW

Coutrot, A., Binetti, N., Harrison, C., Mareschal, I., \& Johnston, A. (2016). Face exploration dynamics differentiate men and women. Journal of Vision, 16(14):16. doi: 10.1167/16.14.16 WwW

Coutrot, A., Hsiao, J. H., \& Chan, A. B. (2017). Scanpath modeling and classification with hidden Markov models. Behavior Research Methods. Advance online publication. doi: 10.3758/ s13428-017-0876-8 WWW

Hall, J. K., Hutton, S. B., \& Morgan, M. J. (2010). Sex differences in scanning faces: Does attention to the eyes explain superiority in facial expression recognition? Cognition and Emotion, 24, 629-637. doi: 10.1080/02699930902906882

Halpern, D. F. (2000). Sex differences in cognitive abilities (3rd ed.) Mahwah, NJ: Lawrence Erlbaum Associates.

Hamann, S., Herman, R. A., Nolan, C. L., \& Wallen, K. (2004). Men and women differ in amygdala response to visual sexual stimuli. Nature Neuroscience, 7, 411-416. doi: 10.1038/nn1208 [wWw

Heisz, J. J., Pattruff, M. M., \& Shore, D. I. (2013). Females scan more than males: A potential mechanism for sex differences in recognition memory. Psychological Science, 24, 1-7. doi: 10.1177/0956797612468281 [ww

Henderson, J. M., Falk, R., Minut, S., Dyer, F. C., \& Mahadevan, S. (2001). Gaze control for face learning and recognition in humans and machines. In: T. F. Shipley \& P. J. Kellman (Eds.), From fragments to objects: Segmentation and grouping in vision (pp. 463-481). Amsterdam, the Netherlands: North Holland.

Henderson, J. M., Williams, C. C., \& Falk, R. J. (2005). Eye movements are functional during face learning. Memory \& Cognition, 33, 98-106. doi: 10.3758/BF03195300 WWW

Herlitz, A., \& Lovén, J. (2013). Sex differences and own-gender bias in face recognition: A meta-analytic review. Visual Cognition, 21, 9-10. doi: 10.1080/13506285.2013.823140

Herlitz, A., \& Rehnman, J. (2008). Sex differences in episodic memory. Current Directions in Psychological Science, 17, 52-58. doi: 10.1111/j.1467-8721.2008.00547.x 
Hewig, J., Trippe, R. H., Hecht, H., \& Miltner, W. H. R. (2008). Gender differences for specific body regions when looking at men and women. Journal of Nonverbal Behavior, 32,67-78. doi: 10.1007/ s10919-007-0043-5

Hickman, L., Firestone, A. R., Beck, F. M., \& Speer, S. (2010). Eye fixations when viewing faces. The Journal of the American Dental Association, 141, 40-46. doi: 10.14219/jada.archive.2010.0019 WWW

Hojjat, M. (2000). Sex differences and perceptions of conflict in romantic relationships. Journal of Social and Personal Relationships, 17, 598-617. doi: 10.1177/0265407500174007

Janik, S. W., Wellens, A. R., Goldberg, M. L., \& Dell'Osso, L. F. (1978). Eyes as the center of focus in the visual examination of human faces. Perceptual and Motor Skills, 47, 857-858. doi: 10.2466/ pms.1978.47.3.857

Kemeny, J. G., \& Snell, J. L. (1960). Finite Markov chain. New York, NY: Van Nostrand.

Loftus, G. R., \& Mackworth, N. H. (1978). Cognitive determinants of fixation location during picture viewing. Journal of Experimental Psychology: Human Perception and Performance, 4, 565-572. doi: 10.1037/0096-1523.4.4.565 |

Pérez-Moreno, E., Romero-Ferreiro, V., \& García-Gutiérrez, A. (2016). Where to look when looking at faces: Visual scanning is determined by gender, expression and tasks demands. Psicológica, 37, 127-150.

Rabiner, L. R. (1989). A tutorial on hidden Markov models and selected applications in speech recognition. Proceedings of the IEEE, 77, 257-287. doi: 10.1109/5.18626
Sæther, L., Van Belle, W., Laeng, B., Brennen, T., \& Øvervoll, M. (2009). Anchoring gaze when categorizing faces' sex: Evidence from eye-tracking data. Vision Research, 49, 2870-2880. doi: 10.1016/j.visres.2009.09.001 WWW

Schurgin, M. W., Nelson, J., Lida, S., Ohira, H., Chiao, J. Y., \& Franconeri, S. L. (2014). Eye movements during emotion recognition in faces. Journal of Vision, 14(13):14. doi: 10.1167/14.13.14 WWW

SMI eye tracking system. http://www.smivision.com $\underline{\underline{W W}}$

Stacey, P. C., Walker, S., \& Underwood, J. D. (2005). Face processing and familiarity: Evidence from eye-movement data. British Journal of Psychology, 96, 407-422. doi: 10.1348/000712605X47422 [WWW

Stumpf, H. (1995). Gender differences in performance on tests of cognitive abilities: Experimental design issues and empirical results. Learning and Individual Differences, 7, 275-287. doi: 10.1016/1041-6080(95)90002-0

Vassallo, S., Cooper, S. L., \& Douglas, J. M. (2009). Visual scanning in the recognition of facial affect: Is there an observer sex difference? Journal of Vision, 9(3):11. doi: 10.1167/9.3.11 WWW

Voyer, D., Voyer, S., \& Bryden, M. P. (1995). Magnitude of sex differences in spatial abilities: A meta-analysis and consideration of critical variables. Psychological Bulletin, 117, 250-270. doi:

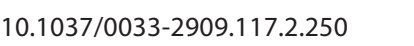

Walker-Smith, G. J., Gale, A., \& Findlay, J. M. (1977). Eye movement strategies involved in face perception. Perception, 6, 313-326. doi: 10.1068/p060313 WWW

RECEIVED 19.10.2016 | ACCEPTED 19.06.2017 